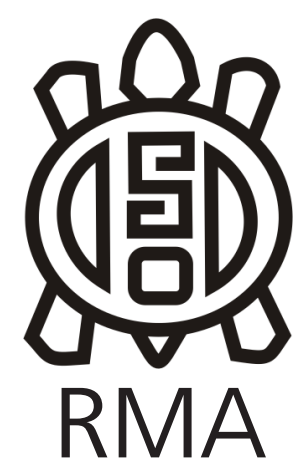

Museología

\title{
Entre el patrimonio y la historia. Transformaciones arquitectónicas de una iglesia puneña ante las lógicas estatales en el siglo XX (Coranzulí, Jujuy, Argentina)
}

\author{
Between heritage and history. Architectural transformations of a \\ church located in Jujuy Highlands at the face of nation state logics in \\ the 20th century (Coranzuli, Jujuy, Argentina)
}

Julieta Barada

CONICET, Instituto Interdisciplinario Tilcara, Facultad de Filosofía y Letras, Universidad de Buenos Aires. E-mail: ju.barada@gmail.com

\begin{abstract}
Resumen
Las arquitecturas religiosas del noroeste argentino construidas principalmente durante el período colonial y en menor medida durante los siglos XIX y XX, conforman una parte significativa de su patrimonio material. Este ha sido registrado y valorado por diferentes organismos nacionales e internacionales. Desde su construcción hasta nuestros días, estas iglesias continuaron formando parte de la vida de las comunidades a las que pertenecen a partir de su significación ritual, la que contempla las prácticas constructivas. Es en el marco de este hacer que se definen diferentes relaciones y sentidos sociales entre las personas, los espacios, los materiales y las técnicas. Así, se trata de un patrimonio que de ningún modo puede ser considerado de un modo inmanente, pero por sobre todo, se trata de procesos sociales que permiten dar cuenta de las transformaciones en las ideas, los intereses y valoraciones construidos por las propias comunidades en su relación con otros agentes. En este artículo nos ocuparemos de analizar las transformaciones experimentadas en la iglesia del pueblo de Coranzulí (Puna de Jujuy) a través del estudio de las acciones realizadas por los pobladores sobre esta a lo largo del siglo XX. Nos apoyaremos en dos tipos de registro: el etnográfico, producido en el trabajo de campo que venimos realizando desde el año 2012, y el documental, basado en las fuentes gráficas y escritas que se produjeron tanto local como institucionalmente en el siglo XX. Consideraremos la relación que las operaciones constructivas realizadas sobre la iglesia han tenido con los distintos procesos de institucionalización de las arquitecturas puneñas a la luz de la inserción de los pastores en las lógicas del estado nacional y, simultáneamente, de sus arquitecturas en los cánones de valoración patrimonial.
\end{abstract}

Palabras Clave: arquitectura eclesiástica, patrimonio, Puna de Jujuy, técnicas constructivas, comunidades pastoriles

\begin{abstract}
The religious architectures of northwestern Argentina, mainly built during the colonial period and, to a lesser extent, during the nineteenth and early twentieth centuries are a significant part of their material heritage. This has been registered and valued by different national and international organizations. From their construction to the present day, these churches continued to be part of the life of the communities which they belong because of their ritual significance, which contemplates constructive practices. In this context, different relationships and social meanings are defined between people, spaces, materials and techniques. It is, then, a patrimony that can not be considered in an immanent way, but it is also about social proceses which allow us to think about idea, interest and by the same communities into their relations with other agents. In this article we will analyze the transformations experienced in the church of Coranzuli (Puna of Jujuy) through the study of the actions carried out on it by the town inhabitants throughout the 20th century. For this, we will rely on two types of record: the ethnographic one, produced from the field work we have been doing since 2012, and the documentary one, based on graphic and written sources produced both locally and institutionally throughout of the 20th century. We will consider the relationship that the constructive operations carried out on the church have had with the different processes of institutionalization of the local architectures that occurred in the context of the insertion of the herders in the logics of the national state and, simultaneously, of their architectures into patrimonial valuation standards.
\end{abstract}

Keywords: ecclesiastical architecture, heritage, Jujuy Highlands, constructive techniques, herders communities

La valoración patrimonial de las arquitecturas del noroeste argentino ha estado históricamente atravesada por una fuerte presencia de los edificios religiosos (Nicolini, 1964; Bolsi y Gutiérrez, 1974; Ascencio et al., 1974;
Sosa y Latina, 2015; entre otros). En general, se trata de iglesias construidas durante el periodo colonial, cuyas arquitecturas dan cuenta de la tensión entre los modelos eclesiásticos europeos y el desarrollo local del culto 
católico, como motor fundamental de la colonización. En este sentido, se trata de un patrimonio que posee un significado histórico singular, en tanto las iglesias han sido agentes materiales centrales en la transformación del territorio, siendo, en general, los primeros edificios institucionales coloniales que se hicieron presentes en el área, germen de muchos de los actuales pueblos ${ }^{1}$. Asimismo, se trata de edificios a través de los cuales a lo largo del tiempo se han ido construyendo relaciones comunitarias y sentidos específicos, comprendidos en el marco de las concepciones espaciales -y por lo tanto sociales- de las comunidades locales. De este modo, cuando nos aproximamos a considerar su valor patrimonial, no podemos pensarlo desde una perspectiva meramente objetual ni mucho menos inmanente sino que, por el contrario, debemos considerar su construcción como tales en una clave dinámica y desde la historia local.Las diferentes operaciones de construcción y reconstrucción a través de las cuales las comunidades ido transformando estas arquitecturas en distintos contextos se constituyen, entonces, como instancias clave para la comprensión de procesos de definición y redefinición socialcomunitarios en los que, muchas veces, los intereses del estado nacional construidos a la luz de sus arquitecturas institucionales resultaron centrales. En este sentido es que en este artículo nos interesa abordar la problemática del rol que posee la técnica y el propio hacer constructivo en la definición de la arquitectura como proceso y objeto de negociación y transformación

\begin{abstract}
${ }^{1}$ Esto se asocia estrechamente -aunque no exclusivamente- con la conformación de los llamados "pueblos de indios" durante la colonia, reducciones indígenas que se extendieron por buena parte del área andina, cuya forma y desarrollo urbano estuvo en línea con las Leyes de Indias. En este marco, Farberman y Boixadós (2006) plantearon que, para que un asentamiento fuera considerado un "pueblo de indios" tenía que cumplir con ciertas condiciones: tener capilla, tener "forma de pueblo", tener otorgadas tierras de comunidad, tener autoridades étnicas reconocidas por la corona y pagar tributo.
\end{abstract}

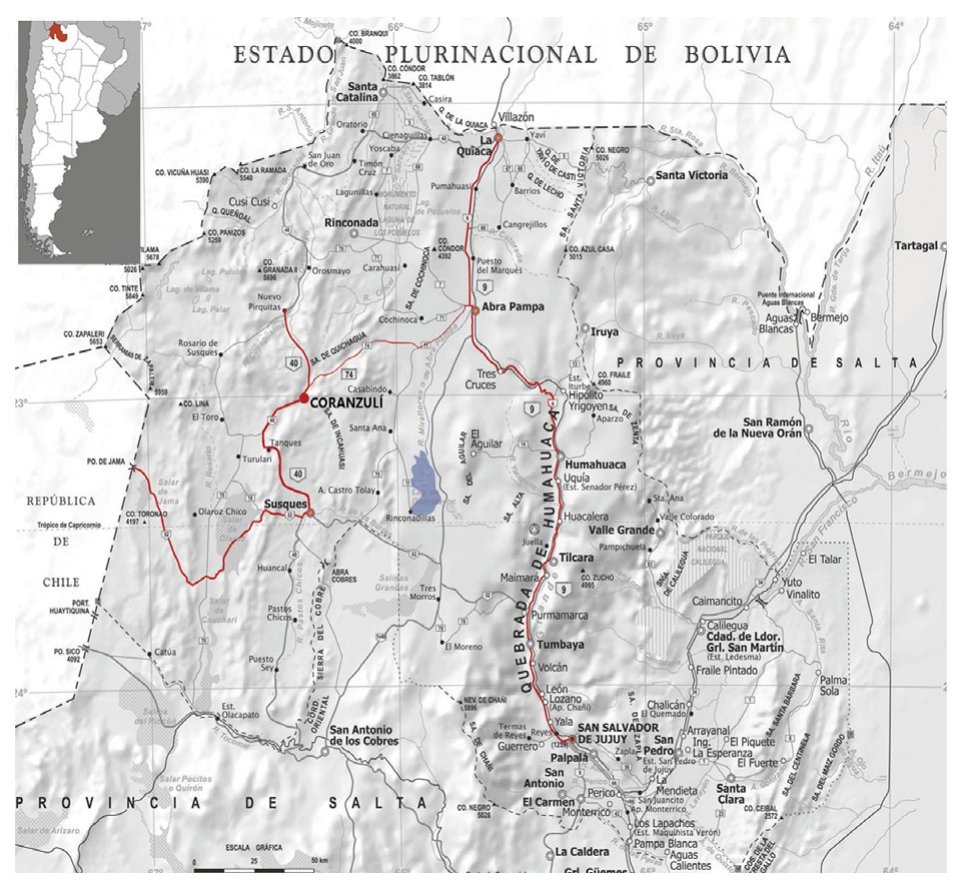

social (Dietler y Herbich, 1998) a partir de un estudio de caso singular como lo es el de la iglesia del poblado de Coranzulí, provincia de Jujuy, Argentina.

Coranzulí es un pueblo localizado en el área puneña de la provincia de Jujuy. Estapuede definirse como "una planicie de alrededor de 12.500.00ha, ubicada por encima de los 3.000m de altura en el extremo noroeste del país" (Reboratti, 2005:33) (Figura 1). Sin embargo, si bien entenderemos a la Puna como la construcción regional muchas veces asociada a la porción del altiplano andino que hoy se localiza dentro de los límites del territorio argentino (Benedetti, 2005), debemos notar que, de acuerdo con Reboratti (2005), sus límites exceden los del territorio nacional, incluyendo parte del sur de Bolivia y del norte chileno, en un área que se extiende por los Andes Centro-Sur. Su población ha estado históricamente dedicada a la actividad pastoril -principalmente de camélidos- con alta movilidad. Es decir que, en línea con las definiciones generales sobre el pastoreo andino (Nuñez y Dillehay, 1995) se trató, históricamente, de una población que sostuvo una movilidad cíclica basada en el desplazamiento conjunto de la unidad doméstica y su hacienda a través de diferentes asentamientos a lo largo del año, de acuerdo con la disponibilidad de pasturas, y que únicamente acudía a los poblados -en este caso al de Coranzulí- para momentos específicos del año.

Sin embargo, para poder comprender el devenir de los pueblos puneños y su arquitectura a lo largo del siglo XX y en particular, sus dinámicas actuales, debemos tener en cuenta la creciente incorporación de otras actividades económico-productivas que han estado en línea con muchas de las políticas e intereses propiciados por los diferentes estados nacionales a los que estos grupos han sido insertos desde el siglo XIX. Así, en el contexto de la Puna argentina, el impacto de la apertura de nuevos mercados laborales -especialmente el de la minería- el incremento del empleo público y el comercio, resultan relevantes para comprender la actualidad del pueblo de Coranzulí. En este sentido, debemos mencionar que su inserción a las estructuras estatales modernas presenta algunas características singulares ya que perteneció sucesivamente a diferentes estados nacionales: en el marco de los procesos independentistas latinoamericanos llevados a cabo durante el siglo XIX, primero formó parte de Bolivia hasta 1879 y luego,

Figura 1. Mapa de la provincia de Jujuy en el que se destacaron la localización de Coranzulí y las principales localidades y rutas que lo vinculan en rojo (Fuente: elaboración de la autora sobre base de mapa de la provincia de Jujuy, Instituto Geográfico Nacional).

Figure 1. Map of the province of Jujuy in which the location of Coranzuli and the main localities and routes that link it in red were highlighted (Source: Author's elaboration based on map of the province of Jujuy, National Geographic Institute). 
durante la Guerra del Pacífico, pasó a formar parte de Chile (Delgado 2003). En 1899, se estableció el límite definitivo entre Chile y Argentina y el sector oriental de las tierras altas atacameñas donde se localiza Coranzulí -que para entonces se denominaba Puna de Atacama-, pasó a formar parte del territorio argentino. Desde 1900 hasta 1943, Coranzulí estuvo incluido en el Territorio Nacional de Los Andes y desde su disolución fue incorporado, con el resto del departamento de Susques, a la provincia de Jujuy (Benedetti, 2005). La inserción de los pastores en las lógicas político-económicas del estado argentino a lo largo del siglo XX tuvo consecuencias en el rol que los pueblos, y el de Coranzulí en particular, ocupa en sus dinámicas cotidianas. En este sentido, sus edificios y en particular aquellos asociados a instituciones que, como la Iglesia Católica, han participado activamente en la definición político-social del estado nacional, se constituyen como estructuras significativas para poder problematizar las dinámicas sociales de su población en relación con las demandas e intereses propiciados por el propio estado.

A continuación, haremos una somera descripción del rol que las iglesias han tenido, históricamente, en la conformación del espacio y en particular el de los pueblos en los Andes para el cual las técnicas constructivas empleadas en su producción, así como los rituales colectivos asociados a estos edificios han sido centrales. Luego, realizaremos una breve aproximación cómo intervinieron los procesos de inserción institucional de Coranzulí en la redefinición de muchas técnicas constructivas a la luz de la construcción de discursos "oficiales" y "académicos" sobre el uso de ciertos materiales como el adobe y la paja en la arquitectura puneña. A partir de lo planteado en estos dos primeros apartados, analizaremos la trayectoria histórica de la iglesia de Coranzulí como caso de estudio en el área puneña, estableciendo relaciones con lo sucedido en casos del área. Finalmente, reflexionaremos acerca del rol de la técnica en la redefinición de sentidos sociales, apuntando al modo en que los intereses locales -aun cuando muchos de estos hayan sido construidos a la luz de sentidos estatales definidos de un modo profundamente asimétrico- operan a la hora de pensar en este tipo de arquitecturas como patrimonio en clave dinámica y compleja.

La estrategia metodológica que emplearemos se apoya en la articulación entre dos tipos de registro: el etnográfico -producido a partir del trabajo de campo que venimos realizando en Coranzulí desde el año 2012y el documental, constituido por las fuentes gráficas y escritas que se produjeron tanto localmente como a nivel institucional a lo largo del siglo $X X$, a las que hemos tenido acceso a través de diferentes reservorios documentales "oficiales" (Archivo General de la Nación y Archivo Histórico de Jujuy) y a través del acceso a fuentes inéditas "locales" (Archivo de ordenanzas de la Comisión
Municipal de Coranzulí y Libro Histórico de la Escuela N³62 de Coranzulí 'Héroes de Malvinas').

\section{Las iglesias en los Andes: conformaciones espaciales y técnicas constructivas como procesos de definición social}

El rol que las iglesias poseen en la definición de las relaciones comunitarias en diferentes grupos pastoriles en los Andes ha sido destacado por diferentes investigadores en contextos diversos. Como ejemplos, podemos mencionar trabajos de Ossio (1981), Urton (1989) y Sendón (2004) en Perú, el de Arnold (1998) en Bolivia, así como el de Tomasi (2011) en Susques, en la Puna jujeña, mucho más cercano a nuestro caso de estudio. Esto se asocia, al menos, a dos cuestiones centrales que debemos considerar cuando nos aproximamos a comprender la significación que estos edificios poseen en clave local: la primera es su rol en la conformación del espacio del pueblo y su ritualidad, en relación con las dinámicas de las familias de pastores en el campo. La segunda tiene que ver con el rol social que poseen las técnicas constructivas en tierra y sus prácticas asociadas en los Andes en general.

En relación con la primera cuestión, debemos considerar a las iglesias como materialidades centrales en la conformación de los pueblos de pastores en los Andes como aquellos sitios a los que las familias, asentadas en el campo y dedicadas al pastoreo, acudían únicamente en momentos puntuales, generalmente rituales. De hecho, en el pionero trabajo de Métraux (1936) sobre los Chipayas de Carangas, el autor señala que el edificio de la Iglesia Católica que se alza en el pueblo es el único edificio común a todos los ayllus. En este sentido, es a partir de la iglesia como núcleo común que se organiza la estructura urbana de un pueblo como lugar del colectivo (Tomasi, 2011). Es por ello que, el análisis de la estructura urbana de este tipo de poblados -incluso cuando estén atravesadas por las lógicas de ordenamiento urbano colonial basadas en las Leyes de Indias- se distingue, en muchos casos, un ordenamiento de las residencias y de los espacios comunes que responde a las relaciones constituidas en el campo entre diferentes grupos de familias y sus asentamientos ${ }^{2}$.

La segunda cuestión, particularmente relevante para los intereses de este artículo, se define en el modo en que estas iglesias han sido construidas a través de diferentes

\footnotetext{
${ }^{2}$ En los trabajos que analizan las relaciones comunitarias de los pastores en el conteo peruano y/o boliviano este tipo de relaciones están asociadas a la idea del ayllu (Fuenzalida, 1972; Arnold, 1998; Allen, 2002; entre otros). En el contexto Argentino, encontramos un ordenamiento socio-espacial que vincula a diferentes grupos de familias de pastores asentadas en el campo a través de la noción de sección. Así, mientras que, por ejemplo, los pastoreos localizados en los alrededores del pueblo de Susques, se organizan en dos secciones -norte y sur- (Tomasi, 2011), en el caso Coranzuleño se distinguen en la actualidad tres secciones -Incahuasi, Agua Delgada y Quebrada Grande- que ordenan las pertenencias y las localizaciones de las unidades domésticas pastoriles en el campo.
} 
estrategias de trabajo comunitario, pero, especialmente, en cómo las técnicas constructivas han formado parte de su trayectoria a lo largo del tiempo a través de diferentes prácticas de construcción y reconstrucción comprendidas en el marco de profundos sentidos rituales. Entre estas, el retechado o repaje de las iglesias cuyas cubiertas habían sido realizadas con guaya -paja y barro- es una de las más significativas, observada en distintos contextos. Uno de los casos estudiados sobre este ritual, es el analizado por Sendón (2004): el wasichakuyen Marcapata, Perú. Por su parte, Tomasi (2011) registró el retechadode la iglesia de Susques, a partir de un trabajo colectivo en el que se involucraban tanto la secciónnorte como la sur, reforzando los lazos establecidos al interior de cada una y también entre ambas, como partes de la conformación del colectivo susqueño. Estas prácticas son uno de los muchos aspectos a través de los cuales es posible considerar la centralidad que posee la arquitectura en los Andes y en particular su aspecto técnico. En este sentido, podemos pensar en el rol social de las técnicas y su definición en el marco de construcciones colectivas singulares, como instancias de negociación de sentidos sociales. Como ha planteado Pfaffemberger: "la gente construye su mundo social usando los recursos sociales y las estructuras que tiene a mano, pero sus actividades modifican las estructuras tanto como estas son reproducidas" (1992:500). En los Andes, la construcción es un proceso que es parte de la vida social de las personas, las familias y las comunidades (Arnold, 1998; Tomasi, 2012; entre otros) y que involucra, también, al universo de acciones desde el cual estos objetos son nombrados, clasificados, categorizados y usados por las personas.

A la luz de esto último, observaremos a continuación cómo a partir de los procesosde valoración de las arquitecturas puneñas producidos tanto desde el ámbito académico como estatal en la Argentina -y en particular en la provincia de Jujuy-, estos modos de hacer, en principio asociados a lazos familiares y comunitarios, comienzan a involucrar otros actores, ideas e intereses que permiten problematizar cómo desde la materialidad se han ido negociando ciertos sentidos sociales ante la acción de los agentes estatales.

\section{La 'doble valoración' de las arquitecturas puneñas}

Los discursos e ideas que se construyeron desde el estado y sus agencias sobre la arquitectura de la Puna se apoyaron, en buena medida, en los relatos de funcionarios que recorrieron el área desde finales del siglo XIX y principios del XX sobre la precariedad de las construcciones (Castro, 2007; Tomasi, 2015). Las consecuencias -incluso alejadas en el tiempo- de los discursos construidos entonces, pueden ser observadas en las valoraciones y clasificaciones que aun al día de hoy se elaboran desde distintos dispositivos del estado -como el censal- sobre las arquitecturas del área. La clasificación del adobe como material de "ínfimo valor" ha sido de hecho, sostenida desde el ámbito oficial desde la Ley
Nacional N 4.167 de 1911 e incluso hasta el día de hoy, en tanto el adobe continúa sin ser reconocido por los organismos oficiales de normalización y certificación ${ }^{3}$ como un material habilitado para la construcción ${ }^{4}$.

Como ha observado Jerez (2013) el aliento a las construcciones "de material" que erradicarían de un modo definitivo los "ranchos de adobe" en la provincia de Jujuy, encuentra su génesis en el pensamiento liberalconservador de la Argentina del orden y el progreso de comienzos del siglo $\mathrm{XX}$, pero se ha sostenido como proyecto político a lo largo de todo el siglo. Aun en este contexto, podemos mencionar que en la actualidad del pueblo de Coranzulí, las viviendas con muros de adobe alcanzan el $95 \%$ de las construcciones. Sin embargo, simultáneamente, otras transformaciones como la de los techados de guaya por el uso de la chapa de zinc o el reemplazo de los pisos de tierra apisonada por cerámicos o alisados de cemento, sí resultan mucho más significativas. Así, podemos notar que aquellas políticas e ideas que en los centros urbanos nacionales y regionales impactaron de un modo mucho más directo, fueron operadas, también, desde aquellos sitios en los que, como Coranzulí, las distancias respecto de los centros de poder eran mayores. Como veremos, el rol que en estos procesos de inserción institucional a través de la arquitectura tuvieron las agencias de los pobladores ha sido central.

Por su parte, si desde el plano técnico, la erradicación de las arquitecturas de tierra por aquellas "de material" fue uno de los ejes que caracterizó a la arquitectura del proyecto civilizatorio de la Argentina de comienzos del siglo para el cual la masiva inmigración europea de finales del siglo XIX ocupó un lugar central, en el plano compositivo estilístico fueron las arquitecturas europeas las que constituyeron la imagen que debía reemplazar al "rancho". Hubo entonces en la Argentina un florecimiento de las arquitecturas académicas y eclécticas, especialmente en lo que respecta al desarrollo de los edificios institucionales e infraestructuras que posibilitaron la consolidación del estado nacional tanto en términos políticos como simbólicos (Liernur, 2008). La Argentina inició un proceso de claro deslinde con el pasado colonial y sus arquitecturas que tuvo a Buenos

\footnotetext{
${ }^{3}$ Se refiere a las Normas IRAM (Instituto Argentino de Normalización y Certificación), organismo desde el que se emiten las normas técnicas de construcción en la Argentina.

${ }^{4}$ Las discusiones en torno a la utilización de este material son, en sí mismo, un complejo campo de estudios cuya referencia excede los intereses de este artículo. Sin embargo, nos interesa clarificar aquí que las ideas e imaginarios en torno a la debilidad de este material constituidas históricamente en el área, distan de las reales posibilidades constructivas del mismo, en el marco de sistemas constructivos coherentes que posibiliten su uso y su efectividad como componente de estructuras portantes incluso en zonas de riesgo sísimico, tal como ha sido intensamente estudiado en el caso peruano (Blondet et al., 2011) y normalizado a través de la norma E-80. En esta misma línea, existen también importantes avances normativos en torno al uso del adobe y la construcción con tierra en general en diferentes países (Cid y Cañas, 2011)
} 
Aires como cabecera, en función de la consolidación de una nación fundamentalmente agro-exportadora, con miras a los mercados industriales -y en particular culturales-, de las grandes capitales europeas (Barada, 2017). De un modo paralelo, debemos considerar las miradas que se construyeron desde el ámbito académico sobre estas mismas arquitecturas, las que guiaron su proceso de valoración patrimonial. En este sentido mencionamos que fueron justamente las iglesias las primeras obras reconocidas en el área puneña como patrimonio. Esto condujo a una revalorización de un conjunto de construcciones locales, principalmente por considerarlas dotadas de un valor "tradicional" que se asocia, estrechamente, al pasado colonial del área concentrado en obras de carácter monumental como las iglesias ${ }^{5}$.

En relación con estas ideas 'oficiales' y 'académicas' que históricamente se construyeron sobre las arquitecturas puneñas es que podemos observar una tensión entre, por un lado, el proceso de transformación de los pueblos que se dio a la luz de la acción estatal y por el otro, el 'rescate' de las arquitecturas locales llevado a cabo desde el ámbito académico, germen del despliegue de las políticas de protección patrimonial en el área. Así, muchas iglesias del noroeste argentino fueron declaradas como Monumentos Históricos Nacionales, pasando a formar parte de los marcos normativos específicos elaborados por la Comisión Nacional de Monumentos, Lugares y Bienes Históricos para su protección. En este sentido, se trata de obras cuya custodia y conservación a lo largo del tiempo depende del estado nacional, en concurrencia con las autoridades locales -ya sean municipales o provinciales- a las que pertenezca por dominio el Bien ${ }^{6}$.

Esto presenta un primer problema en relación con el modo en que las prácticas de construcción y reconstrucción presentadas en el acápite anterior, que hacen a las arquitecturas partícipes de la trayectoria histórica de sus comunidades y sus prácticas, se mantienen o no en el marco de estas políticas. Si bien es preciso notar que la propia noción de patrimonio y por lo tanto sus políticas asociadas, se han ido transformando a lo largo del tiempo en función de constituir una mirada dinámica sobre el patrimonio material que contemple, asimismo, sus aspectos inmateriales (UNESCO, 2003), las prácticas de los diferentes grupos sociales conducen, muchas veces, a acciones sobre los bienes patrimoniales que

\footnotetext{
${ }^{5}$ Este tipo de búsquedas condensaron las producciones de arquitectos como Sacriste, Caveri o Vautier, pero también en publicaciones y relevamientos que se ocuparon de poner de relieve las arquitecturas existentes, en particular las coloniales, tales como "Pueblos de encomienda Jujeña" de Iglesia y Ortiz en la Revista Nuestra Arquitectura entre los años 1963 y 1965; la de Ascencio, Iglesia y Schenone (1974) sobre los casos de Casabindo y Cochinoca, así como los trabajos realizados por Nicolini (1964) en la Quebrada de Humahuaca, sobre el pueblo de Purmamarca y la publicación de Bolsi y Gutiérrez sobre Susques (1974).

${ }^{6}$ Ley N¹2.665. Modificación. Comisión Nacional de Monumentos, de Lugares y de Bienes Históricos. Enero de 2015.
}

transforman partes o incluso la totalidad de sus aspectos a preservar, cuestión que genera tensiones entre los diferentes intereses y agentes que operan sobre el mismo patrimonio.

El segundo problema tiene que ver con el sostenimiento de las miradas 'oficiales' sobre las condiciones precarias de las arquitecturas del área que se da en simultáneo a la progresiva institucionalización del espacio puneño a través de la inserción de la población local en la vida cívica del estado y los mercados laborales capitalistas asociados a este, conduciendo a la transformación de las propias prácticas comunitarias. Entonces, ¿qué otros intereses mediaron en los procesos de construcción y reconstrucción local de las iglesias en la Puna, que exceden a la lógica de 'articulación comunitaria andina'? ¿cómo la introducción de otras ideas arquitectónicas han transformado el hacer constructivo local y, por lo tanto, el rol social que las técnicas y las materialidades tienen para las propias comunidades?

En este sentido es que a continuación nos ocuparemos de un caso singular, como es el de la iglesia de Coranzulí. Esta presenta, al menos dos condiciones diferenciales que nos permiten problematizar estas cuestiones desde una perspectiva amplia. La primera es que, aun cuando, como veremos, se trate de una obra que ha tenido características formales y estilísticas que pueden ser consideradas coloniales, de acuerdo con los documentos que brindan la fecha de su construcción, no se trata de una iglesia de origen colonial sino que su construcción se da, prácticamente, a comienzos del siglo $X X$, en el contexto de la inserción de la Puna de Atacama al estado argentino. La segunda -que se vincula con la primera- es que no se trata de una obra protegida por ninguna legislación ni provincial ni nacional, motivo por el cual las prácticas de construcción y reconstrucción de la iglesia persisten sin restricción a cargo de los propios pobladores, expresando allí, intereses complejos y muchas veces contradictorios.

\section{La iglesia de Coranzulí como caso de estudio}

En línea con las apreciaciones que venimos haciendo acerca de las miradas 'oficiales' y 'académicas' sobre las arquitecturas puneñas, antes de adentrarnos en el devenir de la iglesia de Coranzulí, vale que reproduzcamos un fragmento de una descripción sobre la arquitectura eclesiástica de la Puna de Jujuy, plasmada en un libro inédito de 1961, titulado "El patrimonio de la provincia de Jujuy":

"Aparecen así las primeras iglesias que fueron chozas
de adobe de planta rectangular un poco más grandes
que las usadas por la gente. También de barro eran
los pisos y techos a dos aguas con vigas acopladas
(...) el resultado es siempre una obra rústica y muraria
materializada con el mínimo de recursos, expresión 


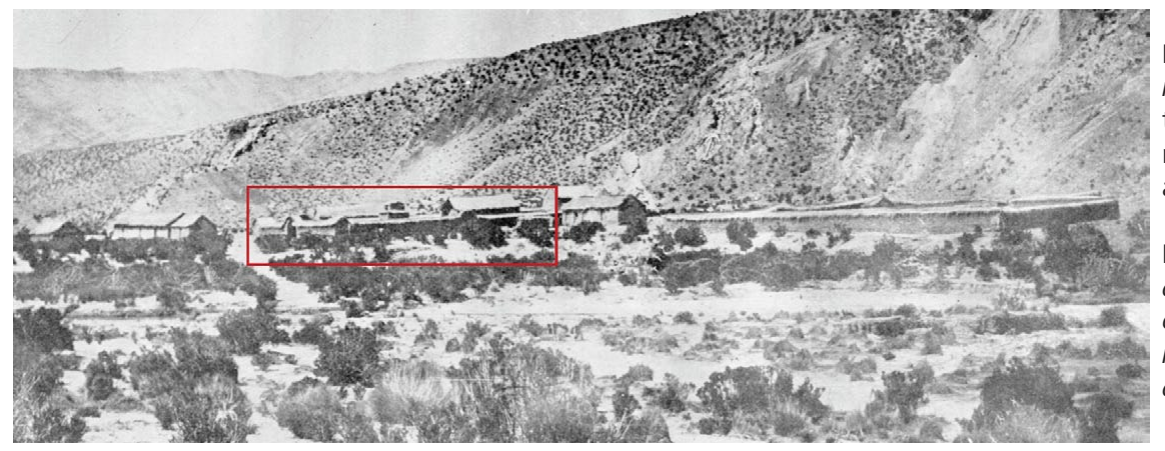

Figura 2. La iglesia de Coranzulí con su atrio y mojón en 1900 (Fuente: recorte de la imagen tomada por Cerri -1903- con indicación en recuadro de la localización de la iglesia y su atrio).

Figure 2. The church of Coranzuli with its atrium and landmark in 1900 (Source: clipping of the image taken by Cerri -1903- with indication in box of the location of the church and its atrium).

regional integrada con el ambiente que respira el deseo de lo auténtico. La arquitectura es parte del suelo al que pertenece." (1961:229).

Como vemos, la clave de lectura sobre las arquitecturas locales, construida a partir de la tensión entre el desprecio y la valoración romántica que planteamos anteriormente entre las diferentes miradas, se expresa aquí en referencia a las arquitecturas eclesiásticas, en general las primeras instituciones 'foráneas' presentes en los pueblos desde la colonia.

A partir del trabajo de campo realizado y de la lectura de las fotografías históricas y actuales de Coranzulí podemos dar cuenta, al menos, de cuatro etapas de construcción visibles en el edificio de la iglesia: (1) la iglesia al momento de la anexión, fotografiada por el Gral. Daniel Cerri -primer gobernador del Territorio Nacional de Los Andes-, en 1900; (2) la modificación sin fecha precisa que puede verse en las fotografías que forman parte del relevamiento realizado por la Academia Nacional de Bellas Artes en 1942 -las fotografías fueron tomadas por Han Mann-; (3) la modificación realizada en 1945, que se observa recién en los registros fotográficos del arquitecto Federico Ortiz, tomados en 1970; (4) la actual.

La primera iglesia, 'piedra fundamental' de Coranzulí Debemos tener en cuenta que para el momento de construcción de la iglesia de Coranzulía finales del siglo XIX, la población local no sólo ya había sido cristianizada, sino que había conocido iglesias coloniales a partir de las relaciones que su población tenía tanto en términos sociales como políticos con Susques y de sus viajes de intercambio a los ayllus del oeste de Atacama, tales como San Pedro o Toconao. Lo propio podemos decir de las relaciones que los coranzuleños tenían con los poblados de Casabindo y Cochinoca (Delgado, 2008), constituidos como pueblos de indios desde su pertenencia a la Gobernación del Tucumán.

\section{Aspectos morfológicos}

La construcción de la primera iglesia en Coranzulí fue motivada y realizada por los propios pobladores que, por entonces, vivían en sus casas en el campo. Este primer edificio estaba conformado por un único recinto rectangular orientado al este, construido con cimientos de piedra y muro de adobe, con techado de guaya (Figura 2). Su distinción respecto de las casas existentes estaba dada por su escala -algo mayor a la de las casas presentes entonces en el poblado- y por la presencia del atrio. Este elemento ha sido característico de las arquitecturas eclesiásticas latinoamericanas desde la colonia (Gisbert y Mesa, 1985). Dentro del atrio se encontraba el mojón, con una altura similar a la de la capilla. En cada una de las esquinas del atrio, se reconoce la presencia de cuatro capillas posas, conocidas también como ermitas ${ }^{7}$. Las capillas posas son capillas semi-abiertas con un altar, que se encuentran localizadas en las cuatro esquinas del atrio, delimitándolo. Las cuatro capillas posas poseen un rol relevante en las prácticas rituales, en tanto operan como instancias de un recorrido procesional en el interior del atrio. Cada una de estas capillas se encuentra orientada a uno de los puntos cardinales, lo que permite observar entre ellas la continuidad de un recorrido. Así, la presencia de estas capillas permite analizar la conformación material de la iglesia y la delimitación del atrio, pero también, y simultáneamente, a la iglesia y al pueblo como lugares de las celebraciones.

\section{Relaciones técnicas, relaciones sociales}

Evidentemente, las condiciones técnicas y morfológicas de esta primera iglesia y el contexto de su producción, nos permite pensar el rol activo que tuvieron los propios pobladores en las decisiones constructivas que aquí se expresan, en negociación con aquellas provenientes de la doctrina católica. Por un lado, la referencia a la tipología de las capillas coloniales en el área andina que se ve claramente representada a partir del posicionamiento del recinto rectangular y su entrada, conformando una única nave. Lo propio sucede a partir de la presencia del atrio y las posas (Gisbert y Mesa, 1985). Por otro lado, la técnica utilizada tanto en la elevación de muros como en la cubierta de la capilla y el alero delantero, remite a las construcciones religiosas construidas en las casas en el campo, como oratorios familiares. Así, el caso de la primera iglesia de Coranzulí nos enfrenta a esta articulación entre la referencia a una arquitectura eclesiástica presente en otros poblados del área y la arquitectura doméstica pastoril como lógica espacial y técnica.

\footnotetext{
7 De acuerdo con Gisbert y Mesa (1985), las "iglesias con atrio y posas" conformaron una tipología extendida en América colonial, particularmente en el siglo XVI. Sin embargo, mientras que su construcción cesó en México para el siglo XVII, en el área andina continuó realizándose incluso hasta el siglo $X X$, lo que formaría parte de la argumentación de su presencia en Coranzulí hacia finales del XIX.
} 


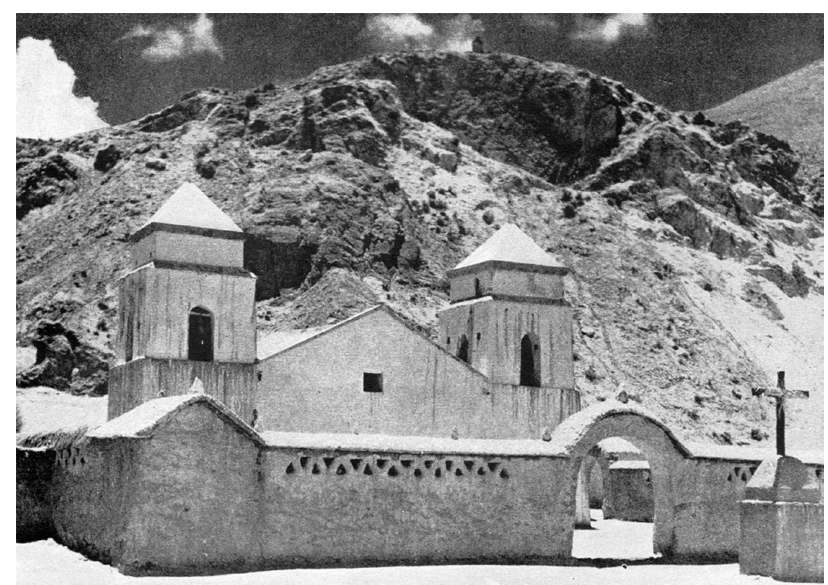

Figura 3. La iglesia de las dos torres en 1942. (Fuente: Academia Nacional de Bellas Artes, 1942).

Figure 3. The church of the two towers in 1942. (Source: National Academy of Fine Arts, 1942).

\section{La iglesia de las dos torres}

La segunda iglesia fue registrada por Hans Mann para la Academia Nacional de Bellas Artes en 1942. Podemos comprender la transformación sustancial que experimentó el edificio para este momento, en el marco de los procesos de crecimiento y transformación de la forma urbana del pueblo que se dieron hacia mitad del siglo XX en el contexto de la disolución del Territorio Nacional de Los Andes, cuando ya estaban instaladas las principales instituciones del estado argentino en el pueblo y este poseía un desarrollo considerable de su forma urbana. Esto resulta significativo para contextualizar el cambio en la morfología de la iglesia desde una arquitectura como la que describimos recién -conformada en la tensión entre la clave doméstica y la clave institucional- hacia una arquitectura que puede ser comprendida claramente en los marcos de referencia constituidos por la academia para definir a las construcciones eclesiásticas andinas coloniales, incluso las presentes en aquellos sitios que han tenido mayor relevancia política y demográfica.

\section{Conformación Morfológica}

La morfología general de esta segunda iglesia, y en particular, de su frente, son completamente distintas a los de la primera (Figura 3). Lo más sobresaliente es su fachada, que se despega del recinto central de la iglesia -o nave- superándolo en altura y ancho. Se trataba de un frente liso, de color blanco, que anulaba el anterior alero frontal que conformaba el acceso a la primera iglesia. Estaba compuesta por un cuerpo central que reproducía, en su remate, la inclinación a dos aguas del techado de la nave, y por dos torres laterales que se elevaban por sobre la altura de la fachada. Mientras que en el centro se localizaba la puerta de acceso a la iglesia y una ventana superior, en las torres se encontraban los campanarios. Este último elemento ha sido otro de los componentes característicos de las arquitecturas religiosas del altiplano (Buschiazzo, 1942)8 (Figura 4).

\footnotetext{
${ }^{8}$ Los campanarios de las iglesias, localizados en las torres, tuvieron ciertas variantes en relación con aquellas que poseían una única torre
}
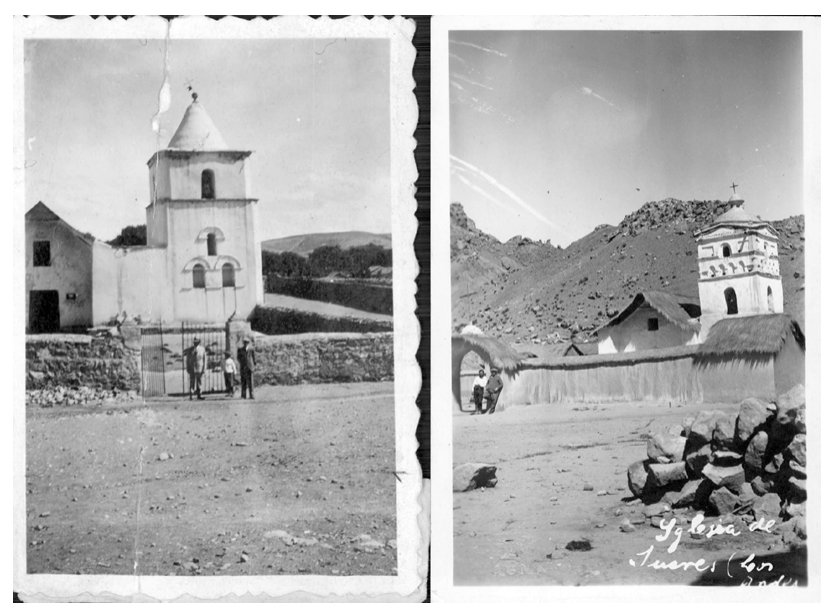

Figura 4. Postales de otras iglesias del área. A la izquierda, imagen de la Iglesia de La Quiaca en 1939 y a la derecha, la de Susques en 1930, ambas con el campanario en una torre lateral (Fuente: Archivo General de la Nación. Departamento de Documentos Fotográficos).

Figure 4. Postcards of other churches in the area. On the left, image of the Church of La Quiaca in 1939 and on the right, that of Susques in 1930, both with the belfry in a side tower (Source: Archivo General de la Nación, Department of Photographic Documents).

Por otra parte, debemos atender a las características del interior de la capilla (Figura 5). En estas se observa la presencia de dos columnas con forma pseudosalomónicas ${ }^{9}$ que rompen con las líneas rectas que expresan las características morfológicas de la fachada de estética colonial. Este elemento, sumado a la ausencia de pinturas murales o retablos de madera que han sido propios de la arquitectura eclesiástica en la colonia (Gutiérrez, 2001), evidencian la tensión que en términos materiales y formales expresa esta iglesia respecto del momento de su construcción.

\section{Relaciones técnicas, relaciones sociales}

La forma que adquiere esta segunda iglesia entre lo andino, lo colonial y el eclecticismo de la arquitectura europea decimonónica, nos enfrenta a una construcción material que lejos de ser la resultante de la imposición de un agente homogéneo y hegemónico se define en la interacción entre las distintas agencias, intereses y sentidos que operaron en el pueblo. Así, el rol que han tenido los pobladores en su propia inserción institucional, en línea con lo que sugerimos a partir del análisis se expresa en las acciones realizadas para la reconstrucción de la iglesia, tanto 'hacia adentro' como 'hacia afuera' del pueblo. En relación con la primera cuestión, podemos volver sobre el registro de los procesos de construcción y reconstrucción de estas arquitecturas a partir del modo en el que estos fueron registrados en el Libro Histórico de la Escuela. Allí, los maestros describieron, con cierto asombro, el proceso de modificación y mantenimiento

lateral (como Susques, Antofagasta de la Sierra), dos torres (como la de Coranzulí o Casabindo) o bien una torre aislada, como sucede en algunos de los poblados del norte chileno (Villalonga, 1942).

${ }^{9}$ Las columnas salomónicas son aquellas de fuste helicoidal, cuyo uso fue extendido en la arquitectura barroca en Europa y en América (ver: Gutiérrez, 2001). 
del edificio de la iglesia:

"Con el objeto de realizar el trabajo de repajar el techo de la iglesia que está en mal estado y mejorar el aspecto de los tapiales que la rodean, todos los vecinos de este extenso distrito de Coranzulí, se reúnen, dando así, muestras de una solidaridad bien entendida, y a la vez, de grandes beneficios en estas tan apartadas regiones. Es interesante y divertido el trabajo por el que se puede apreciar que esta construcción regional que aparentemente está exenta de un algo llamativo y curioso, la gracia que cuando se trata de elevar el prestigio y hasta el orgullo si se quiere de todo el conjunto que ellos forman. Todos, entonces, trabajan por todos, pudiéndose, así, aplicar la vieja y sabida verdad de: 'la unión hace la fuerza'" (Mes de noviembre de 1940 -el subrayado es nuestro-).

La particularidad que posee el evento relatado en relación con la significación que el edificio de la iglesia tiene en el pueblo nos permite plantear, de un modo simultáneo, el entramado de sentidos y lógicas al que nos estamos refiriendo. Como observamos en el relato, la práctica conjunta del repaje del techado y arreglo de la tapia, forma parte de la propia constitución del pueblo como colectivo en el marco de las prácticas y sentidos que han sido registrados, también, en otros sitios de los Andes. Tal como planteamos previamente, las acciones de mantención, reparación y transformación del edificio son también, procesos de definición y redefinición social. A su vez, el mismo relato que presenta la actuación de los pobladores como una práctica de solidaridad interna, la comprende también como un acto en función de elevar el prestigio del conjunto de la población 'hacia afuera'. Más allá del exotismo que guarda la descripción realizada por los maestros, es interesante el registro de esta doble condición que opera en la reparación de la iglesia: por un lado, con el repaje de la nave y por el otro, con el cambio hacia una 'imagen institucional' con el nuevo frente y las dos torres.

\section{La iglesia de torre única}

Las fotografías tomadas por Ortiz en 1970 dan cuenta de una importante modificación: las dos torres fueron reemplazadas por una sola, alterando completamente el frente del edificio (Figura 6).
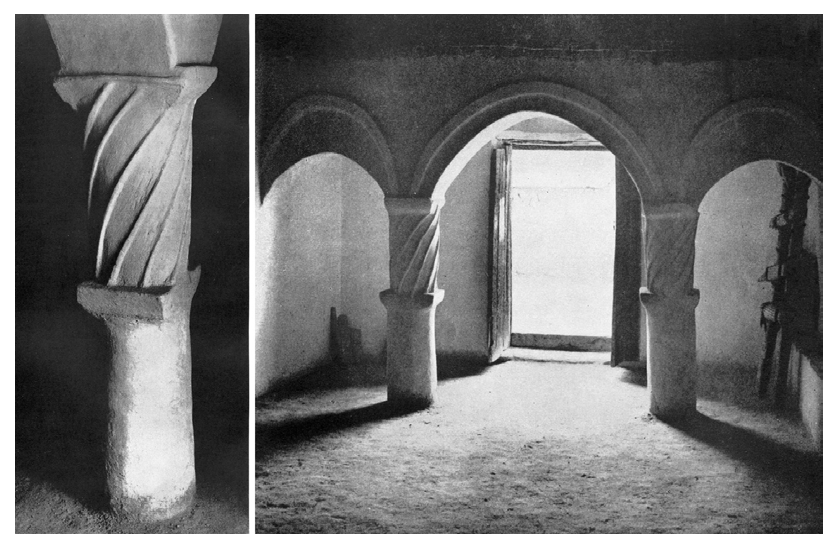

Figura 5 (der.) Interior de la iglesia de Coranzulí, con dos columnas con volutas en el acceso; (izq.) detalle de una de las columnas (Fuente: fotografías de Hans Mann, Academia Nacional de Bellas Artes, 1942).

Figure 5 (right) Interior of the church of Coranzuli, with two columns with scrolls in the access; (left) detail of one of the columns (Source: photographs by Hans Mann, National Academy of Fine Arts, 1942). Conformación morfológica

En sentido horizontal, el nuevo frente estaba dividido en tres partes: la central con el acceso y dos laterales. En cada uno había dos contrafuertes también de piedra que, en principio, estarían operando estructuralmente en el sostenimiento del frente. En sentido vertical, también se puede observar una tripartición de la fachada, con una disminución de su masa superior. El remate, compuesto por el campanario y la cúpula, se encontraba revocado y blanqueado, lo que generó un contraste con el resto del frente. Además de las variaciones en el ancho del frente y la disminución de la masa muraria, la tripartición vertical de la fachada se destaca a partir de cornisas lisas que sobresalen del muro generando un detalle ornamental. En lo que respecta a la nave, esta no fue modificada aunque sí se le han adosado algunos elementos. A cada lado, y en cada extremo de su desarrollo longitudinal, se observa un contrafuerte de piedra que, en principio, supondría una labor estructural en función de sostener los posibles empujes horizontales del muro.

Relaciones técnicas, relaciones sociales Para contextualizar esta transformación de la iglesia, debemos acudir a otros documentos que nos permiten precisar con mayor detalle el momento en el que esta modificación fue realizada. El 11 de noviembre de 1945, el maestro Raúl Torán asentó en el Libro que ese día "todos los vecinos de Coranzulí"10 se congregarían en el pueblo
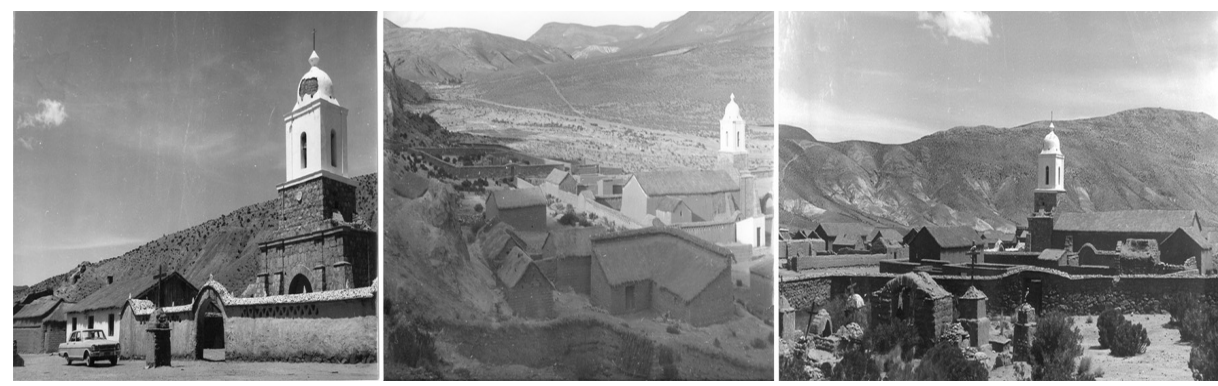

Figura 6. Imágenes de la iglesia de la torre única en 1970. (izq.) frente y vistas desde el sur (centro) y desde el norte (der.) (Fuente: Federico Ortiz, 1970 -(EDODAL-).

Figure 6. Images of the church of the single tower in 1970. (left) front and views from the south (center) and from the north (right) (Source: Federico Ortiz, 1970 - CEDODAL-). 
para la construcción de la torre de la iglesia. Nuevamente, nos encontramos ante una práctica constructiva que implicó la reunión de la comunidad. En este caso, no se trató del ya mencionado repaje sino que de un cambio constructivo que impactó de manera contundente en la imagen de la iglesia. Como puede apreciarse, una práctica constructivo-ritual, como era la de la congregación de los vecinos para la realización de un trabajo colectivo, se articuló con una decisión arquitectónica que no tuvo que ver con el sostenimiento de un edificio en el tiempo sino con una alteración concreta en su morfología, estética y material. Algunas de las personas más ancianas que actualmente viven en el pueblo, recuerdan esta actividad de la cual participaron cuando eran niños o adolescentes. Así, dentro de los relatos que se repiten en relación con ese momento, se recuerda la principal motivación por la cual la torre fue modificada ${ }^{11}$ : toda la comunidad trabajó para cambiar la iglesia... la antigua iglesia era de adobe y no iba a durar...

Este cambio en la iglesia puede ser comprendido, al menos, en dos sentidos asociados al proceso de institucionalización de las arquitecturas localesal cual venimos haciendo referencia: (1) como un cambio formal hacia una estética arquitectónica que dejó atrás el pasado 'colonial' representado en la iglesia de las dos torres, por una arquitectura propia de la clave del progreso que fue promovida por la Generación del '80 y (2) como un cambio técnico que modificó el uso del adobe por el de la piedra, con mortero cementicio.En relación con el primero, podemos notar que, si las dos torres habían sido características de la arquitectura barroca de la América colonial, el cambio hacia la torre central de la Iglesia

Noviembre de 1940. Período Escolar 1940-1941.

${ }^{11}$ Otras versiones locales indican que la iglesia fue modificada debido a que un rayo impactó en una de sus torres. No tenemos datos que nos permitan saber de modo fehaciente cual fue la motivación principal de la transformación de la iglesia. De todos modos, lo que interesa aquí en todo caso es observar cuáles fueron las decisiones estéticas y constructivas que mediaron dichas transformaciones, a la luz de la continuidad de las prácticas locales sobre el edificio y de las preferencias técnicas que comenzaron a emerger entre los actores locales a la luz de los discursos y acciones promovidas por los agentes estatales.

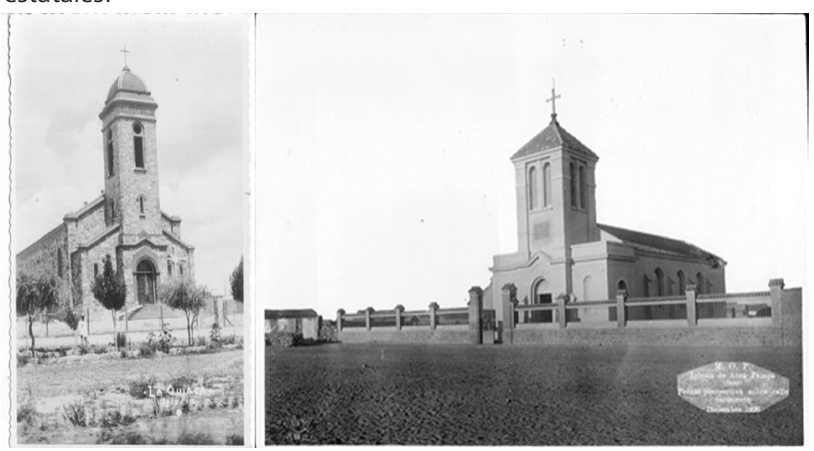

Figura 7. Postal de la Iglesia de La Quiaca en 1939 y frente de la Iglesia de Abra Pampa en 1926 (Fuente: Archivo General de la Nación. Departamento de Documentos Fotográficos).

Figure 7. Postcard of the Church of La Quiaca in 1939 and in front of the Abra Pampa Church in 1926 (Source: Archivo General de la Nación, Department of Photographic Documents). puede asociarse a aquellas otras arquitecturas que, en clave ecléctica y modernista se desarrollaron en los nuevos espacios urbanos. No resulta llamativo entonces que este tipo de frente se vea en la Iglesia de Abra Pampa y posteriormente la nueva iglesia de La Quiaca (Figura 7). Ambas localidades, que habían iniciado sus procesos de crecimiento demográfico y económico con la llegada del ferrocarril en la primera década del siglo $X X, y$ posteriormente con el desarrollo de las rutas nacionales.

El segundo sentido tiene que ver con la cuestión específica de los materiales y su uso. La idea que expresa la población local actual acerca de que la iglesia anterior, construida de adobe, no duraría y que por ese motivo debió ser modificada, se asocia a los discursos y normativas ya citados que se emitieron desde las direcciones de arquitectura del estado acerca de aquellos materiales que debían ser usados o no en las construcciones institucionales, y en particular en las tierras fiscales de los Territorios Nacionales. El uso de la piedra y el cemento comenzó a formar parte del repertorio constructivo de los edificios de las principales instituciones del estado, especialmente aquellos localizados en sitios fronterizos asociados a la defensa del territorio nacional, así como también de las construcciones realizadas en los por entonces incipientes emprendimientos mineros de la provincia.

Podemos destacar, entonces, el rol privilegiado y singular que posee la iglesia como institución que no forma parte directa de la estructura estatal, pero que sin embargo constituye el edificio con mayor visibilidad entre el repertorio edilicio de las instituciones en el pueblo, asociado a su propio origen. La iglesia como institución y como materialidad, es capaz de dar cuenta del rol del pueblo como lugar de las espacialidad pastoril y, simultáneamente, como materialidad a través de la cual la misma comunidad se redefine a sí misma ante los ojos del estado.

\section{La Iglesia en la actualidad}

La última Iglesia a la que nos referiremos es a la actual. A diferencia de los cambios que venimos describiendo, luego de la construcción de la torre central, el edificio no sufrió más modificaciones en la forma y estilo de su frente, aunque sí fue reparado y repintado en distintas oportunidades. Sin embargo, sí se presenta un contundente cambio en sus materiales, con la incorporación del techado de chapa y el revoque de cemento en todo su perímetro, así como en el desarrollo de su atrio, a partir de la incorporación de algunos edificios de servicios.

\section{Conformación morfológica}

En cuanto a los materiales, podemos dar cuenta del reemplazo del techado de guaya, por un techado de chapa de zinc. Para ello, se modificó la pendiente del techo y el ancho de la nave, que creció superando el del frente y 
perdió los contrafuertes observados en la iglesia anterior. A su vez, se reemplazó el adobe en la torre campanario, antes revocada, por el uso de ladrillos cocidos a la vista. Sólo el remate de la torre, con la cúpula, mantuvo el revoque y fue repintado de blanco y celeste. El atrio fue modificado a partir de la incorporación de los edificios del salón de usos múltiples, los baños y la casa parroquial. Todos estos edificios fueron construidos en adobe y revocados con cemento, con cubiertas de chapa de zinc. Particularmente, el salón de usos múltiples avanza sobre el interior de lo que fuera el atrio, modificando su forma y eliminandolas capillas posas de la parte posterior (Figura 8). Por su parte, las localizadas en el frente del atrio, fueron modificadas en su posición, colocándose ambas con su abertura hacia el frente de la iglesia. El muro perimetral fue revocado nuevamente, reemplazando el revoque de barro por otro cementicio. Finalmente, el mojón localizado en el exterior fue eliminado ante la construcción de la plaza del pueblo.

\section{Relaciones técnicas, relaciones sociales}

Estos últimos cambios son especialmente significativos en función del rol histórico que ha tenido la iglesia como lugar de reunión estrechamente vinculado con la actividad ritual de los vecinos. Por un lado, ritualidad asociada a la propia técnica, estrechamente imbricada en el uso de ciertos materiales. El repaje de la iglesia como práctica de activación de ciertos lazos sociales y como práctica constitutiva de la materialidad no está contemplado en esta nueva construcción. Debemos pensar, entonces, en el desplazamiento que existe en la práctica constructiva en la sociabilidad del pueblo, en un contexto de profundas transformaciones económicas y productivas. Como nos ha dicho Fernanda, una de las mujeres más ancianas del pueblo, durante el trabajo de campo: Antes, cuando uno tenía que guayar, uno traía la paja, otro el barro, las mujeres cocinábamos (...) Ahora nadie te ayuda, ni siquiera pagando...

Por otro lado, el rol del atrio como lugar de celebraciones asociado a las capillas posas y al mojón, también se vio interpelado por la inclusión de nuevos edificios que

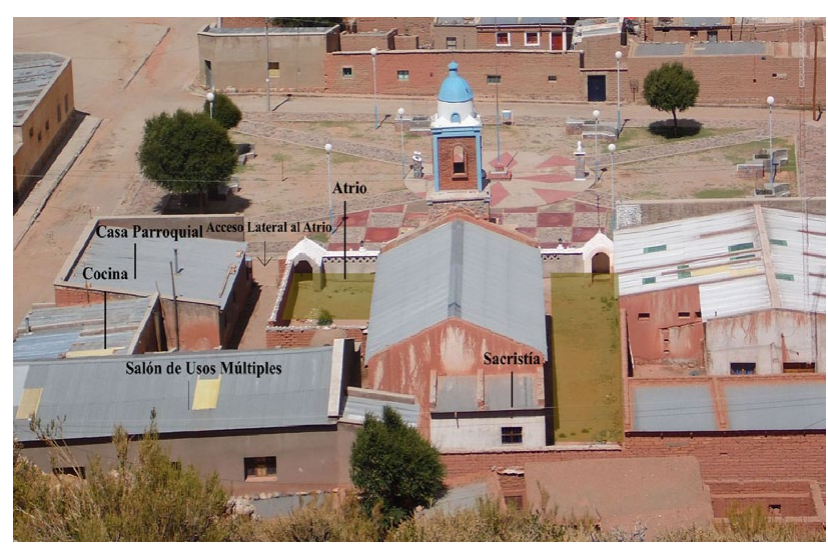

Figura 8. Complejo actual de la iglesia y sus edificios asociados con la modificación del atrio (Fuente: fotografía propia con agregados).

Figure 8. Current complex of the church and its buildings associated with the modification of the atrium (Source: own photograph with additions). albergan funciones sociales asociadas a la iglesia pero no necesariamente relacionadas con lo sagrado. Es en el salón de usos múltiples donde se realizan las reuniones de los grupos de catequesis, los encuentros con fieles de la iglesia de otros pueblos, pero también es el lugar donde se realizan celebraciones y actos escolares e incluso reuniones sociales. Asimismo, debemos notar la contemporaneidad que estas construcciones tienen con la conformación de la plaza de Coranzulí que, a diferencia de lo planteado para los pueblos de indios coloniales, no adquiere su forma actual sino hasta comienzos del siglo $\mathrm{XXI}$, tal como se desprende de las fotografías históricas halladas para la iglesia y el poblado. En este sentido, ciertos recorridos que pudieron estar asociados al atrio y al mojón, se realizan hoy en el espacio extendido del pueblo, con la plaza como centralidad. Esto ocurre, por ejemplo, cada año en la celebración de la Fiesta de la Virgen del Rosario -patrona del pueblo-. La iglesia, su atrio y la plaza conforman el centro desde el cual parten las procesiones, donde se realizan las /uminarias y donde se baila el tradicional baile de cuartos. A su vez, dicho centro es también donde se realiza, en el mismo evento, el "acto cívico", caracterizado por la presencia de autoridades locales y provinciales, y por la realización del desfile cívico-militar del cual participa buena parte de la población coranzuleña (Figura 9) ${ }^{12}$.

\section{Cierre: técnicas foráneas, prácticas locales: intereses divergentes y patrimonios dinámicos}

Como planteamos al comienzo, las iglesias representan una parte significativa del patrimonio arquitectónico de la Puna de la provincia de Jujuy, así como también son una parte central de la historia social de las comunidades que la habitan. Evidentemente, no podemos pensar estas dos condiciones de un modo disociado, en tanto eso implicaría despojar a la arquitectura de su rol central como agente en la construcción social del espacio (Santos, 2006). En este sentido es que nos inclinamos por considerar a la arquitectura, y al patrimonio que esta constituye, como un proceso dinámico e inacabado más que como un objeto inmanente en el tiempo. Pero por sobre todo, consideramos que esto es necesario para poder comprender su rol social, desde las lógicas y los sentidos producidos en las comunidades que los han construido y que los habitan a lo largo del tiempo.

Así, observamos cómo en el caso de las iglesias puneñas, a través de la técnica constructiva y sus prácticas asociadas, se constituyen instancias de construcción y reconstrucción material que son, a su vez, instancias de construcción y reconstrucción social. De esta manera, la técnica y el hacer constructivo deben ser consideradas allí como prácticas colectivas que articulan intereses, muchas veces divergentes dentro de las mismas comunidades, los que las constituyen como agentes históricos. Es a partir de

\footnotetext{
${ }^{12}$ Los usos del espacio durante la celebración de las fiestas patronales es un tema en sí mismo que ha sido desarrollado en otra publicación (Barada, 2016).
} 

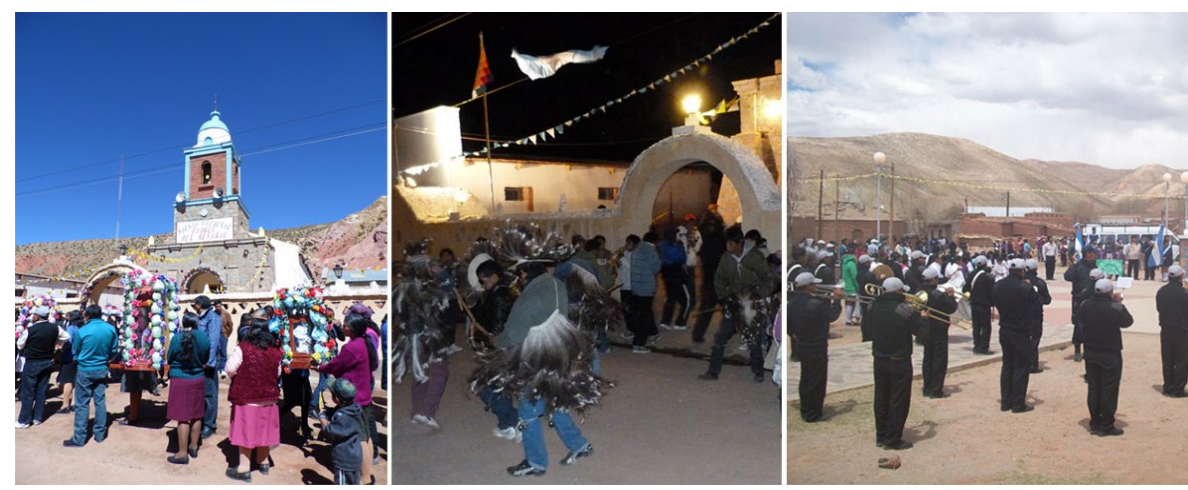

Figura 9. (izq.) Procesión en las Fiestas Patronales de Coranzulí; (centro) baile de los zamilantes en la misma fiesta; y (der.) acto cívico en honor a la Virgen del Rosario en la plaza de Coranzulí(Fuente: fotografías propias).

Figure 9. (left) Procession in the Patron Festivities of Coranzuli; (center) baile de los zamilantes at the same party; and (der.) civic act in honor of the Virgen del Rosario in the Plaza de Coranzulí (Source: own photographs). las elecciones técnicas y materiales que realizaron los pobladores sobre la iglesia de Coranzulíen cada uno de sus cuatro momentos, que pudimos romper con la idea de la aparente imposición de modelos provenientes del campo académico e institucional hegemónico, para comprender el modo en que la propia población local se apropió, discutió y reinterpretó esos mismos sentidos arquitectónicos, aquellos a través de los cuales el propio estado construyó su imagen y procuró constituir un cierto sentido cívico, incluso en una población que permanecía alejada de los centros de poder político. En relación con esto último, el rol que la iglesia ha tenido como edificio significativo para la construcción social de la comunidad lo constituye como un ámbito central para problematizar su propia construcción en relación con procesos de transformación política, económica, y también social, más amplios. En otras palabras, podemos afirmar que, por un lado, las prácticas asociadas a la mantención y reparación del edificio se sostuvieron en el marco de las lógicas locales de cohesión social, en línea con aquellas miradas que buscan reconocer, entonces, a estas mismas prácticas como parte del patrimonio en un sentido dinámico. Pero por otro lado, debemos atender a que las decisiones que en ese mismo proceso han ido tomando los actores locales entorno a la materialidad de la iglesia escapan de aquellas miradas románticas señaladas anteriormente sobre las arquitecturas locales teñidas de una cierta inmanencia en el tiempo, para por el contrario encontrarse con búsquedas y sentidos estéticos provenientes de los actores hegemónicos y sus valoraciones, operadas desde los actores locales en la negociación por su propia construcción ciudadana. En relación con esto último debemos preguntarnos entonces acerca del rol que han tenido no sólo las valoraciones sobre la arquitectura y su materialidad sino aquellos procesos de transformación vinculados a las actividades económico-productivas de la población y su inserción en una economía capitalista. Finalmente, los resultados de las operaciones realizados sobre la iglesia la han despojado de aquellos aspectos que han sido valorados por los organismos provinciales y nacionales en otras capillas andinas en función de su consideración como patrimonio.

A partir de este análisis es que consideramos necesario volver a preguntarnos sobre el modo en que se construyen sentidos patrimoniales sobre la arquitectura que aun cuando procuren considerar a esta de un modo dinámico, se encuentran las más de las vecesenmarcados en políticas y normativas apoyadas en esquemas valorativos que provienen de los saberes expertos y/o institucionales definiendo los objetos y la posibilidad o no de su transformación a lo largo del tiempo. En este sentido, ¿Acaso las operaciones observadas hubieran tenido lugar si una declaratoria patrimonial hubiese involucrado a la iglesia de Coranzulí, como sí sucedió con otros casos del área? Esto implicaría, concretamente, limitar aquellas prácticas asociadas a su construcción y reconstrucción, cuyos sentidos y dinámicas se definen localmente, incluso cuando en su propio hacer operen sentidos promovidos por otros espacios y agentes. Acaso debemos considerar el rol que ha tenido la condición de la iglesia como edificio institucional de relevancia en el pueblo para que la población local experimente allí un cambio de materialidad en consonancia con las miradas que habían sido expresadas por los agentes estatales sobre la durabilidad y calidad de las construcciones, así como aquellas materialidades empleadas en los edificios públicos de otros centros urbanos, mientras que, por otro lado, el adobe sigue siendo el material principalmente elegido para la construcción de arquitecturas domésticas en el pueblo, incluso al día de hoy.

En este sentido es que consideramosnecesario pensar a las políticas patrimoniales desde las propias comunidades a las que pertenecen los edificios, que son la que, finalmente, dan sentido a su propia valoración como tales. Pero por sobre todo, asumir un desafío asociado que requiere revisar la mirada, muchas veces romántica, que el ámbito académico posee sobre estas mismas comunidades -en particular las pastoriles puneñas- para pasar a comprender su acción como agentes históricos, sociales y políticos, cuyos intereses, ideas y materializaciones, escapan, las más de las veces -tal como sucede con la iglesia de Coranzulí- a los que se construyen desde los ámbitos hegemónicos a los que el patrimonio, finalmente, responde.

Así, lejos de ser un monumento que expresa la arquitectura de un único momento histórico definido en función de los períodos y estilos artísticos contemplados en la historiografía 'oficial', la iglesia de Coranzulí encarna, en su propia materialidad, un proceso histórico complejo y heterogéneo cuyos sentidos han sido construidos desde los actores locales.

Tilcara, 18 de Septiembre de 2017. 


\section{Bibliografía}

Arnold, D. (1998). La casa de adobe y piedras del Inka: Género, memoria y cosmos en Qaqachaka. En Arnold, D., D. Jiménez y J. Yapita (Eds.) Hacia un Orden Andino de las Cosas (pp. 31-109). La Paz: Hisbol/ILCA.

Ascencio, M., Iglesia, R. y H. Schenone. (1974). Arquitectura en el altiplano jujeño. Buenos Aires: Librería Técnica CP67.

Barada, J. (2016). Escalas y espacialidades: interacciones desde la celebración de las fiestas patronales en un pueblo puneño. Coranzulí, provincia de Jujuy, Argentina. Revista Geopantanal (20), 09-32.

Barada, J. (2017).Un pueblo es un lugar. Materialidades y movilidades de los pastores puneños ante las lógicas del estado. Coranzulí, Jujuy, Argentina (Tesis de Doctorado). Facultad de Filosofía y Letras, Universidad Buenos Aires, Buenos Aires. Inédita.

Benedetti, A. (2005). Un territorio andino para un país pampeano. Geografía histórica del Territorio de Los Andes (1900-1943) (Tesis de Doctorado). Facultad de Filosofía y Letras, Universidad Buenos Aires, Buenos Aires. Inédita.

Blondet, M., J. Vargas, N. Tarque y C. Iwaki (2011). Construcción sismorresistente en tierra: la gran experiencia contemporánea de la Pontificia Universidad Católica del Perú. Informes de la Construcción (63), 41-50.

Bolsi, A. y R. Gutiérrez. (1974). Susques. Notas sobre la evolución de un pueblo puneño, Documentos de Arquitectura Nacional (2), 14-29.

Buschiazzo, M.A. (1942). Arquitectura religiosa popular en la Argentina. Buenos Aires.

Castro, H. (2007). Otras miradas, otros lugares. Los relatos de viajeros en la construcción de la Puna argentina. En Zusman, P., Lois, C., Castro, H. (Eds.). Viajes y geografías. Exploraciones, turismo y migraciones en la construcción de lugares (pp. 93-113). Buenos Aires: Prometeo.

Cid, J. y M. Cañas (2011) Las normativas de construcción con tierra en el mundo. Informes de la Construcción (63), 159-169.

Delgado, F (2003) El Territorio Nacional de Los Andes. Creación, límites, objetivos económicos del Estado nacional, reacciones de sus actores sociales y problemática por la tenencia de 19001905. Ponencia presentada en IX Jornadas Interescuelas de Departamentos de Historia. Córdoba, Argentina.

Delgado, F. (2008). La ciudadanía en el sector norte del Territorio Nacional de Los Andes (Susques). Perspectiva indígena perspectiva estatal, 1900-1905. Enluorno, G. y E. Crespo (Coords.) Nuevos Espacios. Nuevos problemas. Los territorios nacionales. Neuquén: Educo-Universidad Nacional del Comahue-Universidad Nacional de la Patagonia San Juan Bosco Cehepyc.

Dietler, M y I. Herbich.(1998).Habitus, Techniques, Style: An Integrated Approach to the Social Understanding of Material Culture and Boundaries. En Stark, M (Ed.) The Archaeology of Social Boundaries. (pp. 232-263). Washington, D.C.: Smithsonian Institution Press.

Fuenzalida, F. (1982). La estructura de la comunidad de indígenas tradicional. AA.VV, El campesino en el Perú, 61-104. Lima: Instituto de Estudios Peruanos.

Gisbert, T. y J. Mesa. (1985). Arquitectura Andina. Historia y análisis. La Paz: Colección Arzans y Vela. Embajada de España en Bolivia.

Gutiérrez, R. (2001). Repensando el Barroco americano. Arquitextos, Vitruvius 2(10). Recuperado de: http://www.vitruvius.com.br/ revistas/read/arquitextos/02.019/819

Jerez, M. (2013). El problema de la vivienda en Jujuy durante los gobiernos populares (1945-1962). San Salvador de Jujuy:EdiUnju.

Liernur, J.F. (2008). Arquitectura en la Argentina del Siglo XX. La construcción de la modernidad. Buenos Aires: Fondo Nacional de las Artes.

Nicolini, A. (1964). El Poblado y La Iglesia de Purmamarca. Nuestra Arquitectura (412), 29-36.

Ossio, J. (1981). La estructura social de las comunidades andinas. EnHistoria del PerúllI. (pp. 203-377). Lima: Editorial J. Mejía Vaca.

Pfaffenberger, B. (1992).Social Anthropology of Technology. Annual Review of Anthropology (21), 491-516.

Reboratti, C. (2005). Situación ambiental en las Ecorregiones Puna y Altos Andes. En La situación ambiental en la Argentina (pp. 32-51). Buenos Aires: Fundación Vida Silvestre.

Santos, M. (2006). A Naturaleza do Espaço. San Pablo: Editora da Universidade de São Paulo.

Sendón. P. F. (2004). El wasichakuy de Marcapata. Ensayo de interpretación de una 'costumbre andina'. Revista Andina(39), 51-71.

Sosa, M. y S. Latina. (2015). Atlas de las técnicas de construcción con tierra y su producción en Argentina. Tucumán:CRIATiC.

Tomasi, J. (2011). Geografías del pastoreo. Territorios, movilidades y espacio doméstico en Susques (provincia de Jujuy). (Tesis de Doctorado). Facultad de Filosofía y Letras, Universidad de Buenos Aires, Buenos Aires. Inédita.

Tomasi, J. (2012). Lo cotidiano, lo social y lo ritual en la práctica del construir. Aproximaciones desde la arquitectura puneña (Susques, provincia de Jujuy, Argentina).Apuntes, 5(1), 7-21.

Tomasi, J. (2015). Una expresión del atraso' La arquitectura de la Puna de Atacama en las descripciones de viajeros, científicos y funcionarios a comienzos del siglo XX. Estudios Sociales del NOA. Nueva Serie (15), 83-112.

UNESCO (2003). Convención para la salvaguarda del patrimonio cultural inmaterial. París: UNESCO.

Urton, G. (1988). La arquitectura pública como texto social: La historia de un muro de adobe en Pacariqtambo, Perú (1915-1985). RevistaAndina (6), 225-263.

Villalonga, A. (1942). Ramificaciones del camino de la Quebrada de Humahuaca y de camino de los Incas. Cuaderno I bis. Documentos de Arte Argentino.Buenos Aires: Publicaciones de la Academia Nacional de Bellas Artes. 